\title{
Preoperative predicted risk does not fully explain the association between red blood cell transfusion and mortality in coronary artery bypass grafting
}

\author{
Gaetano Paone, MD, MHSA, ${ }^{a}$ Robert Brewer, MD, MHSA, ${ }^{a}$ Patricia F. Theurer, BSN, ${ }^{\mathrm{c}}$ Gail F. Bell, MSN, ${ }^{\mathrm{c}}$ \\ Chad M. Cogan, MS, ${ }^{\mathrm{b}}$ and Richard L. Prager, MD, ${ }^{\mathrm{d}}$ for the Michigan Society of Thoracic and \\ Cardiovascular Surgeons
}

\begin{abstract}
Objective: Perioperative red blood cell transfusion is associated with increased morbidity and mortality after coronary artery bypass grafting (CABG). Whether transfusion is a cause of these outcomes or serves as a surrogate for a high-risk patient population remains uncertain. This retrospective study tested the hypothesis that increased preoperative risk profile of patients receiving transfusion would explain the relationship between red blood cell transfusion and operative mortality in isolated CABG.
\end{abstract}

\begin{abstract}
Methods: A total of 31,818 patients undergoing isolated CABG were entered into a statewide collaborative database between January 2006 and June 2010. With the Society of Thoracic Surgeons risk calculator, patient cohorts were stratified into 4 groups by predicted risk of mortality (PROM) of less than $2 \%, 2 \%$ to $5 \%$, more than $5 \%$ to $10 \%$ and more than $10 \%$. The association between blood transfusion and mortality was tested at each stratum with a $\chi^{2}$ test. A Breslow-Day test for homogeneity of odds ratios was used to test whether the 4 odds ratios of the strata were similar, and a Cochran-Mantel-Haenszel test was used to test the association between blood transfusion and mortality while controlling for predicted risk mortality strata.
\end{abstract}

Results: In all, 17,720 (55.7\%) of all patients were transfused during the hospitalization. Incidence of transfusion increased stepwise with risk level; $93.3 \%$ of patients with PROM greater than $10 \%$ received blood. Operative mortality was $2.1 \%$ overall, $0.6 \%$ among the $44.3 \%$ of patients who were not transfused, and $3.3 \%$ in the transfused group (odds ratio, $6.19 ; P<.0001$ ). The association between blood transfusion and mortality was significant within each predicted risk stratum. Increased mortality associated with transfusion was statistically equivalent across all predicted risk strata $(P=.1778)$. The association between blood transfusion and mortality for all patients lessened somewhat when controlling for PROM (odds ratio, 2.99 vs 6.19), yet remained highly significant $(P<.0001)$.

Conclusions: The association between red blood cell transfusion and mortality after CABG is highly significant and independent of increased preoperative risk status. The correlation persists after controlling for increased PROM. (J Thorac Cardiovasc Surg 2012;143:178-85)

Perioperative red blood cell transfusion is associated with increased morbidity and both short- and long-term mortality after coronary artery bypass grafting (CABG) ${ }^{1-5}$ The many articles reporting these relationships have repeatedly documented that the demographic risk profile of patients

From the Division of Cardiac Surgery ${ }^{\mathrm{a}}$ and Department of Public Health Sciences, Henry Ford Hospital, Detroit, Mich; the Michigan Society of Thoracic and Cardiovascular Surgeons Quality Collaborative, ${ }^{\mathrm{c}}$ East Lansing, Mich; and the Section of Cardiac Surgery, ${ }^{\text {d }}$ Department of Surgery, University of Michigan, Ann Arbor, Mich.

Disclosures: Authors have nothing to disclose with regard to commercial support.

Read at the 91st Annual Meeting of The American Association for Thoracic Surgery, Philadelphia, Pennsylvania, May 7-11, 2011.

Received for publication May 2, 2011; revisions received Aug 1, 2011; accepted for publication Sept 15, 2011; available ahead of print Oct 19, 2011.

Address for reprints: Gaetano Paone, MD, MHSA, Division of Cardiac Surgery, Henry Ford Hospital, 2799 West Grand Blvd, B1439, Detroit MI, 48202 (E-mail: gpaone1@hfhs.org).

0022-5223/\$36.00

Copyright (c) 2012 by The American Association for Thoracic Surgery doi: $10.1016 /$ j.jtcvs.2011.09.015 who receive transfusion differs significantly from those who do not. Consequently, whether transfusion is a cause of these adverse outcomes or serves as a surrogate marker for a patient population at higher risk remains uncertain. This retrospective study tests the hypothesis that an increased preoperative risk profile of patients receiving transfusion would explain the relationship between red blood cell transfusion and operative mortality in isolated CABG.

\section{MATERIALS AND METHODS \\ Patient Population}

The Michigan Society of Thoracic and Cardiovascular Surgeons Quality Collaborative is a multidisciplinary group consisting of all 33 hospitals that perform adult cardiac surgery in the state of Michigan. ${ }^{6}$ All programs use the Society of Thoracic Surgeons (STS) data collection form and submit data on a quarterly basis to both the STS database and the collaborative. Data managers meet quarterly for ongoing education and training in data abstraction and outcomes reporting. In addition, there are scheduled conference calls and web-based seminars that focus specifically on issues 


\section{Abbreviations and Acronyms \\ $\mathrm{CABG}=$ coronary artery bypass grafting \\ $\mathrm{PROM}=$ predicted risk of mortality \\ STS $=$ Society of Thoracic Surgeons}

related to institutional quality initiatives or data definitions. Data audits are conducted annually to ensure data integrity. Seventy-six data elements are audited from a random sample of 20 cases at each center. These data elements include STS morbidity and mortality risk model variables, intraoperative data, and process and outcome measures. Data managers are instructed to correct data abstraction errors and submit corrected data to the STS during the next data harvest. For consistency, all audit visits are conducted by the same 2 quality collaborative nurses. The last yearly audit, completed in 2010 , revealed $97 \%$ accuracy with $100 \%$ documentation of 30-day follow-up.

Between January 2006 and June 2010, a total of 31,818 patients undergoing isolated CABG were entered into the statewide collaborative database and form the basis of this report. Patients undergoing both on-pump and off-pump procedures were included. Through application of the STS risk calculator, ${ }^{7}$ patient cohorts were stratified into 4 groups according to predicted risk of mortality (PROM) of less than $2 \%, 2 \%$ to $5 \%$, greater than $5 \%$ to $10 \%$, and greater than $10 \%$. The STS risk model for CABG predicts the risk of operative mortality and morbidity on the basis of 30 preoperative patient demographic and clinical variables and can adjust for case mix when comparing outcomes across institutions with different patient populations. $^{7}$

\section{Statistical Analyses}

The response rates for each of 23 preoperative patient characteristics that were considered clinically relevant were summarized. The relationships between these variables and both blood transfusion and mortality were assessed in separate univariate analyses. Quantitative variables were compared with 2-sided 2-sample $t$ tests. Categoric variables were compared between groups with $\chi^{2}$ tests for association.

Because the distributions of PROM observed for both transfused and nontransfused patients were severely skewed, a 2-sided Mann-WhitneyWilcoxon test was used to test whether there was a statistically significant difference in the distribution of PROM between the 2 groups. A $\chi^{2}$ test was used to test whether there was an association between mortality and blood transfusion. An odds ratio and a $95 \%$ confidence interval were computed to estimate the strength of the association. The association between blood transfusion and mortality was tested at each PROM stratum with a $\chi^{2}$ test. A Breslow-Day test for homogeneity of odds ratios was used to test whether the 4 odds ratios among the strata were similar, and a CochranMantel-Haenszel was used to test the association between blood transfusion and mortality while controlling for PROM strata.

A Cochran-Armitage trend test was used to test for a trend in transfusion rate by increasing ordinal PROM strata. PROM was used as a predictor of transfusion in a simple linear regression model. The effects of each risk stratum in reference to the less than $2 \%$ PROM group were assessed with the Wald $\chi^{2}$ estimates.

A propensity score analysis was performed in a 2-step process to adjust statistically for group differences in preoperative characteristics of patients who received transfusion versus patients who did not. In step 1, the propensity score was obtained by calculating the predicted probabilities in a multiple logistic regression on predicting transfusion. The model included 17 preoperative variables that had significant relationships with both transfusion and mortality according to univariate testing. Several other variables were excluded because of limited responses. Patient records missing 1 or more responses for any of the 17 variables used in the model were excluded, reducing the final sample size to 29,526 . In step 2, the strength of blood transfusion in predicting mortality was then tested in another multiple logistic regression model after adjustment for the propensity score.

Finally, as a sensitivity analysis, an additional multiple logistic regression model to test for a relationship between transfusion and mortality was constructed. This model had as predictors, in addition to blood transfusion, each of the 17 individual preoperative variables originally used to build the propensity score.

Statistical analyses were performed with SAS statistical software (version 9.2; SAS Institute, Inc, Cary, NC).

\section{RESULTS}

Preoperative characteristics, including univariate comparisons for both transfusion status and mortality, are listed in Table 1. The large majority of studied variables were significantly related to both outcomes $(P<.001)$. Preoperative aspirin use within 5 days of surgery was the only variable studied not significantly associated with either transfusion status $(P=.690)$ or mortality $(P=.298)$. A total of 17,720 patients $(55.7 \%)$ received red blood cell transfusions during the index hospitalization. This transfusion rate is comparable to the rate of $55.0 \%$ from the STS database in a similar time frame. ${ }^{8}$

The incidence of transfusion increased stepwise with risk level; $45 \%$ of patients at the lowest $(<2 \%$ PROM) risk stratum were transfused, and this rate increased to $93.3 \%$ for patients with PROM greater than $10 \%$ (Table 2). Every PROM stratum had a significantly higher transfusion rate in reference to the lowest $(<2 \%$ PROM) group, and this trend was significant $(P<.0001)$. PROM for the $44.3 \%$ of patients not transfused was $1.3 \%$, compared with $3.3 \%$ for the transfused group $(P<.0001)$.

Operative mortality was $2.1 \%$ for the entire group. There was a significant association between blood transfusion and mortality; $0.6 \%$ of those patients not receiving transfusion died, compared with a mortality of $3.3 \%$ in the transfused group (odds ratio, 6.19; $P<.0001$ ). The association between blood transfusion and mortality was also significant within each PROM stratum (Table 3). Overall, there was no significant difference between odds ratios when comparing them across the PROM strata $(P=.1778)$. The association between blood transfusion and mortality for all patients decreased when controlling for the PROM (odds ratio, 2.99 vs 6.19) yet remained highly significant $(P<.0001)$.

Sixteen of the 17 preoperative variables in a multiple logistic regression model were statistically significant predictors of blood transfusion. Although left main coronary disease was not significant, it was still considered in the propensity model because it had a significant association with mortality (Table 4). After adjustment for group differences built into the propensity score, blood transfusion remained a highly significant predictor of mortality (odds ratio, $2.88 ; P<.001)$. Finally, a multiple logistic regression model 
TABLE 1. Univariate analyses on blood transfusion and mortality by preoperative characteristics

\begin{tabular}{|c|c|c|c|c|c|c|}
\hline Preoperative characteristic & $\begin{array}{l}\text { No transfusion } \\
(N=14,098)\end{array}$ & $\begin{array}{l}\text { Transfusion } \\
(\mathbf{N}=\mathbf{1 7 , 7 2 0})\end{array}$ & $P$ value & $\begin{array}{c}\text { Alive } \\
(\mathbf{N}=\mathbf{3 1}, \mathbf{1 5 8})\end{array}$ & $\begin{array}{c}\text { Dead } \\
(\mathbf{N}=660)\end{array}$ & $P$ value \\
\hline Body surface area $\left(\mathrm{m}^{2}\right.$, mean $\left.\pm \mathrm{SD}\right)$ & $2.1 \pm 0.2$ & $2.0 \pm 0.3$ & $<.001$ & $2.1 \pm 0.3$ & $2.0 \pm 0.3$ & $<.001$ \\
\hline Weight $(\mathrm{kg}$, mean $\pm \mathrm{SD})$ & $94.8 \pm 19.4$ & $84.6 \pm 19.5$ & $<.001$ & $89.2 \pm 20.0$ & $83.5 \pm 22.3$ & $<.001$ \\
\hline Age $(y$, mean $\pm S D)$ & $62.2 \pm 10.2$ & $67.4 \pm 10.6$ & $<.001$ & $65.0 \pm 10.7$ & $70.7 \pm 10.8$ & $<.001$ \\
\hline Ethnicity (no.) & & & $<.001$ & & & $<.001$ \\
\hline Asian & $75(1 \%)$ & $162(1 \%)$ & & $231(1 \%)$ & $6(1 \%)$ & \\
\hline Black & $697(5 \%)$ & $1652(10 \%)$ & & $2266(8 \%)>T$ & $83(13 \%)$ & \\
\hline White & $12,160(91 \%)$ & $14,404(86 \%)$ & & $26,048(88 \%)$ & $516(83 \%)$ & \\
\hline Hispanic & $57(0 \%)$ & $85(1 \%)$ & & $140(0 \%)$ & $2(0 \%)$ & \\
\hline Native American & $36(0 \%)$ & $33(0 \%)$ & & $68(0 \%)$ & $1(0 \%)$ & \\
\hline Other & $324(2 \%)$ & $400(2 \%)$ & & $710(2 \%)$ & $14(2 \%)$ & \\
\hline Sex (no.) & & & $<.001$ & & & $<.001$ \\
\hline Female & $1934(14 \%)$ & $6946(39 \%)$ & & $8613(28 \%)$ & $267(40 \%)$ & \\
\hline Male & $12,163(86 \%)$ & $10,774(61 \%)$ & & $22,544(72 \%)$ & $393(60 \%)$ & \\
\hline Ejection fraction $(\%$, mean $\pm \mathrm{SD})$ & $51.9 \% \pm 11.4 \%$ & $50.0 \% \pm 13.1 \%$ & $<.001$ & $51.0 \% \pm 12.4 \%$ & $45.4 \% \pm 14.3 \%$ & $<.001$ \\
\hline Last hematocrit $(\%$, mean \pm SD $)$ & $40.9 \% \pm 4.4 \%$ & $36.5 \% \pm 5.2 \%$ & $<.001$ & $38.6 \% \pm 5.3 \%$ & $36.3 \% \pm 5.4 \%$ & $<.001$ \\
\hline Operative status (no.) & & & $<.001$ & & & $<.001$ \\
\hline Elective & $5866(42 \%)$ & $5447(31 \%)$ & & $11,179(36 \%)$ & $134(21 \%)$ & \\
\hline Emergency & $310(2 \%)$ & $963(5 \%)$ & & $1180(4 \%)$ & $93(15 \%)$ & \\
\hline Urgent & $7918(56 \%)$ & $11,257(64 \%)$ & & $18,766(60 \%)$ & $409(64 \%)$ & \\
\hline Smoker, current or recent (no.) & & & $<.001$ & & & .028 \\
\hline No & $9482(70 \%)$ & $12,853(76 \%)$ & & $21,846(73 \%)$ & $489(77 \%)$ & \\
\hline Yes & $4066(30 \%)$ & $4117(24 \%)$ & & $8037(27 \%)$ & $146(23 \%)$ & \\
\hline Peripheral arterial disease (no.) & & & $<.001$ & & & $<.001$ \\
\hline No & $12,382(88 \%)$ & $14,208(80 \%)$ & & $26,138(84 \%)$ & $452(68 \%)$ & \\
\hline Yes & $1716(12 \%)$ & $3510(20 \%)$ & & $5018(16 \%)$ & $208(32 \%)$ & \\
\hline Cerebrovascular disease (no.) & & & $<.001$ & & & $<.001$ \\
\hline No & $12,558(89 \%)$ & $14,311(81 \%)$ & & $26,396(85 \%)$ & $473(72 \%)$ & \\
\hline Yes & $1539(11 \%)$ & $3407(19 \%)$ & & $4759(15 \%)$ & $187(28 \%)$ & \\
\hline Chronic lung disease (no.) & & & $<.001$ & & & $<.001$ \\
\hline No & $11,618(82 \%)$ & $13,793(78 \%)$ & & $24,977(80 \%)$ & $434(66 \%)$ & \\
\hline Mild & $1642(12 \%)$ & $2408(14 \%)$ & & $3929(13 \%)$ & $121(18 \%)$ & \\
\hline Moderate & $523(4 \%)$ & $885(5 \%)$ & & $1358(4 \%)$ & $50(8 \%)$ & \\
\hline Severe & $314(2 \%)$ & $633(4 \%)$ & & $892(3 \%)$ & $55(8 \%)$ & \\
\hline Diabetes (no.) & & & $<.001$ & & & .101 \\
\hline No & $9026(64 \%)$ & $9989(56 \%)$ & & $18,641(60 \%)$ & $374(57 \%)$ & \\
\hline Yes & $5072(36 \%)$ & $7731(44 \%)$ & & $12,517(40 \%)$ & $286(43 \%)$ & \\
\hline Hypertension (no.) & & & $<.001$ & & & .121 \\
\hline No & $2351(17 \%)$ & $2220(13 \%)$ & & $4490(14 \%)$ & $81(12 \%)$ & \\
\hline Yes & $11,747(83 \%)$ & $15,497(87 \%)$ & & $26,665(86 \%)$ & $579(88 \%)$ & \\
\hline Renal failure with dialysis (no.) & & & $<.001$ & & & $<.001$ \\
\hline No & $13,449(99 \%)$ & $16,458(97 \%)$ & & $29,319(98 \%)$ & $588(91 \%)$ & \\
\hline Yes & $106(1 \%)$ & $572(3 \%)$ & & $623(2 \%)$ & $55(9 \%)$ & \\
\hline $\begin{array}{l}\text { Previous coronary artery bypass } \\
\text { grafting (no.) }\end{array}$ & & & $<.001$ & & & $<.001$ \\
\hline No & $13,728(97 \%)$ & $16,961(96 \%)$ & & $30,085(97 \%)$ & $604(92 \%)$ & \\
\hline Yes & $370(3 \%)$ & $759(4 \%)$ & & $1073(3 \%)$ & $56(8 \%)$ & \\
\hline Heart failure (no.) & & & $<.001$ & & & $<.001$ \\
\hline No & $13,085(93 \%)$ & $14,555(82 \%)$ & & $27,216(87 \%)$ & $424(64 \%)$ & \\
\hline Yes & $1007(7 \%)$ & $3165(18 \%)$ & & $3936(13 \%)$ & $236(36 \%)$ & \\
\hline Myocardial infarction (no.) & & & $<.001$ & & & $<.001$ \\
\hline No & $7548(56 \%)$ & $8128(48 \%)$ & & $15,476(52 \%)$ & $200(32 \%)$ & \\
\hline Yes & $5999(44 \%)$ & $8843(52 \%)$ & & $14,407(48 \%)$ & $435(69 \%)$ & \\
\hline Myocardial infarction timing & & & .018 & & & .934 \\
\hline $1-7 \mathrm{~d}$ & $2441(39 \%)$ & $3783(41 \%)$ & & $6043(40 \%)$ & $181(40 \%)$ & \\
\hline
\end{tabular}


TABLE 1. Continued

\begin{tabular}{|c|c|c|c|c|c|c|}
\hline Preoperative characteristic & $\begin{array}{c}\text { No transfusion } \\
(\mathrm{N}=\mathbf{1 4 , 0 9 8})\end{array}$ & $\begin{array}{c}\text { Transfusion } \\
(\mathrm{N}=\mathbf{1 7 , 7 2 0})\end{array}$ & $P$ value & $\begin{array}{c}\text { Alive } \\
(\mathbf{N}=\mathbf{3 1 , 1 5 8})\end{array}$ & $\begin{array}{c}\text { Dead } \\
(\mathbf{N}=660)\end{array}$ & $P$ value \\
\hline Any other time & $3788(61 \%)$ & $5424(59 \%)$ & & $8942(60 \%)$ & $270(60 \%)$ & \\
\hline $\begin{array}{c}\text { Adenosine diphosphate receptor } \\
\text { inhibitors within } 5 \mathrm{~d} \text { (no.) }\end{array}$ & & & $<.001$ & & & .006 \\
\hline No & $6985(92 \%)$ & $7528(84 \%)$ & & $14,240(88 \%)$ & $273(83 \%)$ & \\
\hline Yes & $634(8 \%)$ & $1367(15 \%)$ & & $1945(12 \%)$ & $56(17 \%)$ & \\
\hline Aspirin within $5 \mathrm{~d}$ (no.) & & & .690 & & & .298 \\
\hline No & $2753(20 \%)$ & $3497(20 \%)$ & & $6110(20 \%)$ & $140(22 \%)$ & \\
\hline Yes & $11,027(80 \%)$ & $13,849(80 \%)$ & & $24,371(80 \%)$ & $505(78 \%)$ & \\
\hline Left main disease $\geq 50 \%$ (no.) & & & $<.001$ & & & $<.001$ \\
\hline No & $9671(69 \%)$ & $11,543(65 \%)$ & & $20,821(67 \%)$ & $393(60 \%)$ & \\
\hline Yes & $4427(31 \%)$ & $6175(35 \%)$ & & $10,335(33 \%)$ & $267(40 \%)$ & \\
\hline Diseased vessels (no.) & & & $<.001$ & & & $<.001$ \\
\hline 1 & $746(5 \%)$ & $503(3 \%)$ & & $1232(4 \%)$ & $17(3 \%)$ & \\
\hline 2 & $3104(22 \%)$ & $3239(18 \%)$ & & $6243(20 \%)$ & $100(15 \%)$ & \\
\hline 3 & $10,214(73 \%)$ & $13,947(79 \%)$ & & $23,619(76 \%)$ & $542(82 \%)$ & \\
\hline
\end{tabular}

adding blood transfusion to those 17 preoperative variables found to be significant predictors of transfusion and mortality, again demonstrated a significant relationship between transfusion and mortality (odds ratio, 3.05; $P<.001$; Table 5).

\section{DISCUSSION}

The association between blood transfusion and morbidity and mortality in CABG is well established. In a series of more than 8000 patients who underwent a variety of cardiac surgical procedures, Murphy and colleagues ${ }^{2}$ reported that red blood cell transfusion was strongly linked to increased rates of stroke, myocardial infarction, renal impairment, infections, prolonged stay, increased hospital costs, and early and late mortality. Koch and associates ${ }^{3}$ reported comparable relationships between transfusion and increased morbidity and operative mortality and concluded, "Perioperative red cell transfusion is the single factor most reliably associated with increased risk of postoperative morbid events after isolated coronary artery bypass grafting." Other reports that have focused on specific outcomes, including acute renal injury, pulmonary morbidity, and heart failure, have shown similar results. ${ }^{9-13}$ Each of these series exemplifies the available literature regarding outcomes related to blood transfusion in cardiac surgery; invariably, transfused patients have higher rates of morbidity and mortality than those not transfused. Also common to each of these reports, however, is the fact that the demographic characteristics of the transfused group significantly differ from those not receiving transfusion, with transfused patients seeming to represent a cohort at higher risk. Consequently, it remains uncertain whether transfusion is the causal event leading to these worse outcomes or rather is a marker for a sicker patient population that is more likely to undergo transfusion for many reasons.

We initiated this analysis with the hypothesis that a difference in the preoperative risk profile of patients receiving transfusion would explain the increased risk associated with transfusion. Demographic factors identified as significant predictors of transfusion were essentially the same as those that predicted mortality, lending support to our premise. The STS risk calculator ${ }^{7}$ for CABG was used to stratify the study population into 4 cohorts specifically on the basis of the preoperative PROM, and the PROM for the total group of transfused patients in this study population was indeed significantly greater than that for nontransfused patients.

An additional finding of this study was the significant association between PROM strata and the rate of transfusion. As PROM increased, so too did the likelihood of transfusion. Forty-five percent of patients in the lowest risk stratum

TABLE 2. Association between incidence of blood transfusions and predicted risk of mortality groups

\begin{tabular}{|c|c|c|c|c|c|c|}
\hline PROM & Total (no.) & Not transfused (no.) & Transfused (no.) & Odds ratio & $\mathbf{9 5} \%$ Confidence interval & $P$ value* \\
\hline$<2 \%$ & $21,957(69.0 \%)$ & $12,074(55.0 \%)$ & $9883(45.0 \%)$ & Reference & & - \\
\hline $2 \%-5 \%$ & $6722(21.1 \%)$ & $1656(24.6 \%)$ & $5066(75.4 \%)$ & 3.74 & $3.51-3.98$ & $<.0001$ \\
\hline$>5 \%-10 \%$ & $2079(6.5 \%)$ & $297(14.3 \%)$ & $1782(85.7 \%)$ & 7.33 & $6.46-8.31$ & $<.0001$ \\
\hline$>10 \%$ & $1060(3.3 \%)$ & $71(6.7 \%)$ & $989(93.3 \%)$ & 17.01 & $13.35-21.68$ & $<.0001$ \\
\hline
\end{tabular}

PROM, Society of Thoracic Surgeons predicted risk of mortality. *Probability based on a Wald $\chi^{2}$ statistic for group effects. Overall probability based on a Cochran-Armitage trend test, $<.0001$. 
TABLE 3. Association between blood transfusions and mortality over predicted risk of mortality groups

\begin{tabular}{|c|c|c|c|c|c|c|c|}
\hline & \multicolumn{3}{|c|}{ Mortality } & \multirow[b]{2}{*}{ Transfused } & \multirow[b]{2}{*}{ Odds ratio } & \multirow[b]{2}{*}{$\mathbf{9 5} \%$ Confidence interval } & \multirow[b]{2}{*}{$P$ value* } \\
\hline & No. & All & Not transfused & & & & \\
\hline All patients & 31,818 & $660(2.1 \%)$ & $77(0.6 \%)$ & $583(3.3 \%)$ & 6.19 & $4.88-7.86$ & $<.0001$ \\
\hline PROM $<2 \%$ & $21,957(69.0 \%)$ & $160(0.7 \%)$ & $39(0.3 \%)$ & $121(1.2 \%)$ & 3.83 & $2.66-5.49$ & $<.0001$ \\
\hline PROM $2 \%-5 \%$ & $6722(21.1 \%)$ & $198(3.0 \%)$ & $27(1.6 \%)$ & $171(3.4 \%)$ & 2.11 & $1.40-3.18$ & .0003 \\
\hline PROM >5\%-10\% & $2079(6.5 \%)$ & $146(7.0 \%)$ & $8(2.7 \%)$ & $138(7.7 \%)$ & 3.03 & $1.47-6.25$ & .0016 \\
\hline PROM >10\% & $1060(3.3 \%)$ & $156(14.7 \%)$ & $3(4.2 \%)$ & $153(15.5 \%)$ & 4.15 & $1.29-13.35$ & .0098 \\
\hline
\end{tabular}

PROM, Society of Thoracic Surgeons predicted risk of mortality. *Probability based on a $\chi^{2}$ test for association.

received blood; this increased sequentially to rates of $75.4 \%$ at a risk level of $2 \%$ to $5 \%, 85.7 \%$ at the greater than $5 \%$ to $10 \%$ level, and $93.3 \%$ in the group with a PROM of greater than $10 \%$. This finding also supports the idea that the relationship between transfusion and outcomes is at least to some degree dependent on patientspecific characteristics.

Consistent with other studies, patients receiving blood transfusion had an operative mortality that was significantly higher than that among those not transfused. This relationship was significant for the study population as a whole and within each of the 4 individual risk strata. Controlling for PROM in the model diminished but did not fully explain the strength of the relationship. The odds ratio was reduced, but only from 6.19 to 2.99 , and the association remained highly significant. The association between transfusion and mortality was additionally tested with the propensity score analysis and a sensitivity analysis, with similar findings for each model (odds ratios, 2.88 and 3.05, respectively). These results strongly suggest an independent effect of blood transfusion on outcome and therefore only partially support our hypothesis.

TABLE 4. Multivariable predictors of blood transfusion used in propensity score analysis (model size $=\mathbf{2 9 , 5 2 6 )}$

\begin{tabular}{|c|c|c|c|c|c|}
\hline Variable & Effect & Adjusted odds ratio & Confidence interval & Overall $P$ value & $P$ value \\
\hline Body surface area $\left(\mathrm{m}^{2}\right)$ & & 0.119 & $0.073-0.193$ & - & $<.001$ \\
\hline Weight (kg) & & 1.009 & $1.003-1.015$ & - & .005 \\
\hline Patient age (y) & & 1.039 & $1.036-1.042$ & - & $<.001$ \\
\hline \multirow[t]{6}{*}{ Ethnicity } & & & & $<.001$ & \\
\hline & Asian vs white & 1.53 & $1.13-2.06$ & & .006 \\
\hline & Black vs white & 1.78 & $1.60-1.98$ & & $<.001$ \\
\hline & Hispanic vs white & 1.27 & $0.87-1.85$ & & .225 \\
\hline & Native American vs white & 0.79 & $0.47-1.34$ & & .376 \\
\hline & Other vs white & 1.11 & $0.94-1.31$ & & .220 \\
\hline Sex & Female vs male & 2.84 & $2.63-3.07$ & - & $<.001$ \\
\hline Ejection fraction $(\%)$ & & 0.991 & $0.989-0.994$ & - & $<.001$ \\
\hline \multirow[t]{3}{*}{ Operative status } & & & & $<.001$ & \\
\hline & Emergency vs elective & 3.65 & $3.11-4.27$ & & $<.001$ \\
\hline & Urgent vs elective & 1.46 & $1.38-1.55$ & & $<.001$ \\
\hline Smoker, current or recent & Yes vs no & 0.83 & $0.78-0.89$ & - & $<.001$ \\
\hline Peripheral artery disease & Yes vs no & 1.37 & $1.27-1.48$ & - & $<.001$ \\
\hline Cerebrovascular disease & Yes vs no & 1.35 & $1.25-1.46$ & - & $<.001$ \\
\hline \multirow[t]{4}{*}{ Chronic lung disease } & & & & $<.001$ & \\
\hline & Mild vs no & 1.10 & $1.02-1.19$ & & .020 \\
\hline & Moderate vs no & 1.19 & $1.05-1.36$ & & .008 \\
\hline & Severe vs no & 1.29 & $1.10-1.51$ & & .002 \\
\hline Renal failure with dialysis & Yes vs no & 3.78 & $2.98-4.79$ & - & $<.001$ \\
\hline Congestive heart failure & Yes vs no & 1.87 & $1.71-2.05$ & - & $<.001$ \\
\hline Myocardial infarction & Yes vs no & 1.11 & $1.05-1.18$ & - & $<.001$ \\
\hline $\begin{array}{l}\text { Previous coronary artery } \\
\text { bypass grafting }\end{array}$ & Yes vs no & 1.74 & $1.51-2.01$ & - & $<.001$ \\
\hline Left main disease & Yes vs no & 0.96 & $0.91-1.02$ & - & 0.172 \\
\hline \multirow[t]{3}{*}{ No. of diseased vessels } & & & & $<.001$ & \\
\hline & 3 vs 1 & 2.24 & $1.95-2.58$ & & $<.001$ \\
\hline & 2 vs 1 & 1.68 & $1.45-1.95$ & & $<.001$ \\
\hline
\end{tabular}


TABLE 5. Predictability of blood transfusion on mortality with adjustment for preoperative associated variables $($ model size = 29,526)

\begin{tabular}{|c|c|c|c|c|c|}
\hline Variable & Effect & Adjusted odds ratio & Confidence interval & Overall $P$ value & $P$ value \\
\hline Blood transfusion & Yes vs no & 3.05 & $2.34-3.98$ & - & $<.001$ \\
\hline Body surface area $\left(\mathrm{m}^{2}\right)$ & & 0.243 & $0.085-0.693$ & - & .008 \\
\hline Weight $(\mathrm{kg})$ & & 1.016 & $1.003-1.030$ & - & .014 \\
\hline Patient age (y) & & 1.042 & $1.032-1.052$ & - & $<.001$ \\
\hline \multirow[t]{6}{*}{ Ethnicity } & & & & .033 & \\
\hline & Asian vs white & 1.71 & $0.74-3.94$ & & .210 \\
\hline & Black vs white & 1.54 & $1.19-1.99$ & & .001 \\
\hline & Hispanic vs white & 0.77 & $0.19-3.18$ & & .716 \\
\hline & Native American vs white & 0.80 & $0.11-6.01$ & & .828 \\
\hline & Other vs white & 1.13 & $0.64-2.00$ & & .671 \\
\hline Sex & Female vs male & 1.00 & $0.81-1.24$ & - & .984 \\
\hline Ejection fraction $(\%)$ & & 0.988 & $0.982-0.995$ & - & $<.001$ \\
\hline \multirow[t]{3}{*}{ Operative status } & & & & $<.001$ & \\
\hline & Emergency vs elective & 3.84 & $2.81-5.26$ & & $<.001$ \\
\hline & Urgent vs elective & 1.32 & $1.07-1.63$ & & .011 \\
\hline Smoker, current or recent & Yes vs no & 0.92 & $0.74-1.15$ & - & .470 \\
\hline Peripheral artery disease & Yes vs no & 1.49 & $1.23-1.80$ & - & $<.001$ \\
\hline Cerebrovascular disease & Yes vs no & 1.36 & $1.12-1.66$ & - & .002 \\
\hline \multirow[t]{4}{*}{ Chronic lung disease } & & & & $<.001$ & \\
\hline & Mild vs no & 1.55 & $1.24-1.95$ & & $<.001$ \\
\hline & Moderate vs no & 1.62 & $1.17-2.25$ & & .004 \\
\hline & Severe vs no & 2.57 & $1.87-3.51$ & & $<.001$ \\
\hline Renal failure with dialysis & Yes vs no & 2.43 & $1.72-3.51$ & - & $<.001$ \\
\hline Congestive heart failure & Yes vs no & 1.74 & $1.42-2.12$ & - & $<.001$ \\
\hline Myocardial infarction & Yes vs no & 1.36 & $1.12-1.65$ & - & .002 \\
\hline $\begin{array}{l}\text { Previous coronary artery } \\
\text { bypass grafting }\end{array}$ & Yes vs no & 2.32 & $1.70-3.16$ & - & $<.001$ \\
\hline Left main disease & Yes vs no & 1.04 & $0.87-1.24$ & - & .644 \\
\hline \multirow[t]{3}{*}{ No. of diseased vessels } & & & & .378 & \\
\hline & 3 vs 1 & 1.02 & $0.59-1.74$ & & .954 \\
\hline & 2 vs 1 & 0.86 & $0.49-1.52$ & & .599 \\
\hline
\end{tabular}

This investigation is subject to the limitations inherent in any nonrandomized, retrospective, observational study design. The variability of transfusion rates as well as the indications for transfusion at both the institutional and individual surgeon level were not evaluated. Potential differences in outcome related to either the timing or volume of transfusion were not analyzed. The database contains no information regarding the storage duration of transfused blood. Finally, because we used the STS risk algorithm to stratify patients into defined risk groups, it is possible that variables not included in that model could significantly affect the relationships reported here.

Strengths of this study are the multicenter collaborative source and the large data set used in the analysis. Although all large multicenter databases suffer from concerns regarding data completeness and accuracy, audits undertaken annually give us confidence in the accuracy and integrity of the data used to produce this report. ${ }^{14-16}$ The most recently completed audit of the Michigan Society of Thoracic and Cardiovascular Surgeons database revealed 97\% overall agreement and 100\% confirmation of 30-day follow-up.
Furthermore, the comparable rates for transfusion and mortality between these data and data from the STS database tend to support the generalizability of these results.

Much has been written about the possible mechanisms by which perioperative red blood cell transfusion may in fact be harmful. Issues related to immunosuppressive and inflammatory effects and to poor oxygen delivery and red blood cell deformity related to storage time and conditions have been well summarized in a number of publications and are beyond the scope of this discussion. ${ }^{2,17-21}$

Our results confirm a significant association between blood transfusion and increased mortality after CABG that is independent of preoperative risk status. Although we cannot unequivocally attribute these differences to transfusion alone, the results should provide further rationale for the aggressive implementation of blood conservation measures to limit the use of blood transfusion in coronary surgery. Future investigations should evaluate potential differences in outcome related to transfusion triggers, intraoperative versus postoperative transfusion, and the volume of blood transfused. 


\section{References}

1. Moskowitz DM, McCullough JN, Shander A, Klein JJ, Bodian CA, Goldweit RS, et al. The impact of blood conservation on outcomes in cardiac surgery: is it safe and effective? Ann Thorac Surg. 2010;90:451-9.

2. Murphy GJ, Reeves BC, Rogers CA, Rizvi SI, Culliford L, Angelini GD. Increased mortality, postoperative morbidity, and cost after red blood cell transfusion in patients having cardiac surgery. Circulation. 2007;116:2544-52.

3. Koch CG, Li L, Duncan AI, Mihaljevic T, Cosgrove DM, Loop FD, Starr NJ, et al. Morbidity and mortality risk associated with red blood cell and bloodcomponent transfusion in isolated coronary artery bypass grafting. Crit Care Med. 2006;34:1608-16.

4. Engoren MC, Habib RH, Zacharias A, Schwann TA, Riordan CJ, Durham SJ. Effect of blood transfusion on long-term survival after cardiac operation. Ann Thorac Surg. 2002;74:1180-6.

5. Koch CG, Li L, Duncan AI, Duncan AI, Mihaljevic T, Loop FD, et al. Transfusion in coronary artery bypass grafting is associated with reduced long-term survival. Ann Thorac Surg. 2006;81:1650-7.

6. Prager RL, Armenti FR, Bassett JS, Bell GF, Drake D, Hanson EC, et al. Cardiac surgeons and the quality movement: the Michigan experience. Semin Thorac Cardiovasc Surg. 2009;21:20-7.

7. Society of Thoracic Surgeons STS Risk calculator and models. Available at: http://www.sts.org/quality-research-patient-safety/quality/risk-calculator-andmodels. Accessed April 25, 2011.

8. Michigan STS Custom Report. 2010 Harvest 1: report 30. Durham (NC): Duke Clinical Research Institute, Duke University Medical Center; produced July 2010 .

9. Karkouti K, Wijeysundera DN, Yau TM, Callum JL, Cheng DC, Crowther M, et al. Acute kidney injury after cardiac surgery: focus on modifiable risk factors. Circulation. 2009;119:495-502.

10. Habib RH, Zacharias A, Schwann TA, Riordan CJ, Engoren MC, Durham SJ, et al. Role of hemodilutional anemia and transfusion during cardiopulmonary bypass in renal injury after coronary revascularization: implications on operative outcome. Crit Care Med. 2005;33:1749-56.

11. Koch C, Li L, Figueroa P, Mihajevic T, Svensson L, Blackstone EH. Transfusion and pulmonary morbidity after cardiac surgery. Ann Thorac Surg. 2009;88: 1410-8.

12. Surgenor SD, DeFoe GR, Fillinger MP, Likosky DS, Groom RC, Clark C, et al. Intraoperative red blood cell transfusion during coronary artery bypass graft surgery increases the risk of postoperative low-output heart failure. Circulation. 2006;114(1 Suppl):I43-8.

13. Habib RH, Zacharias A, Schwann TA, Riordan CJ, Durham SJ, Shah A. Adverse effects of low hematocrit during cardiopulmonary bypass in the adult: should current practice be changed? J Thorac Cardiovasc Surg. 2003;125:1438-50.

14. Brown ML, Lenoch JR, Schaff HV. Variability in data: the Society of Thoracic Surgeons National Adult Cardiac Surgery Database. J Thorac Cardiovasc Surg. 2010;140:267-73.

15. Grunkemeier GL, Furnary AP. Data variability and validity: the elephant in the room. J Thorac Cardiovasc Surg. 2010;140:273-5.

16. Shahian DM, Edwards F, Grover FL, Jacobs JP, Wright CD, Prager RL, et al. The Society of Thoracic Surgeons National Adult Cardiac Database: a continuing commitment to excellence. J Thorac Cardiovasc Surg. 2010;140:955-9.

17. Spiess BD. Choose one: damned if you do/damned if you don't! Crit Care Med. 2005;33:1871-4.

18. Rawn JD. Blood transfusion in cardiac surgery: a silent epidemic revisited. Circulation. 2007;116:2523-4.

19. van Straten AH, Soliman Hamad MA, van Zundert AA, Martens EJ, ter Woorst JF, de Wolf AM, et al. Effect of duration of red blood cell storage on early and late mortality after coronary artery bypass grafting. J Thorac Cardiovasc Surg. 2011;141:231-7.

20. Koch CG, Li L, Sessler DI, Figueroa P, Hoeltge GA, Mihajevic T, Blackstone EH. Duration of red-cell storage and complications after cardiac surgery. $N$ Engl J Med. 2008;358:1229-39.

21. Rao SV, Califf RM. Is old blood bad blood? Am Heart J. 2010;159:710-2.

\section{Discussion}

Dr Gus J. Vlahakes (Boston, Mass). First, I want to thank the authors for providing the manuscript of the article and the presentation well in advance of this meeting and also the Association for the privilege of discussing this. Second, I have no disclosures to reveal pertinent to this discussion.

Dr Paone, you and your coauthors have examined an important subject of current interest that has been the subject of several studies in the last decade, the important ones being quoted in your written article. With the Michigan Society of Thoracic and Cardiovascular Surgeons database, you have analyzed data from more than 30,000 patients, and the power of this study rests in the large number of patients derived from an audited, well-designed cardiac surgical database. This foundation lends tremendous statistical power and for all practical purposes eliminates the risk of type II statistical errors.

The study confirms that after risk adjustment, red blood cell transfusion remains a significant risk factor for complications and mortality. The potential weakness of this study, however, as you yourselves have pointed out, is the fact that the database may not necessarily contain the data critical for analyzing transfusion decisions, and in particular transfusion timing, relative to the occurrence of complications, which themselves may mandate transfusion.

As with previous studies on this subject, several important questions arise. Looking across the participating institutions, including your own, do you have any preliminary analysis of transfusion triggers for any institutions with fixed criteria such as hematocrit? If so, were guidelines tailored to age, preoperative risk profile, or other parameters? Importantly, can you determine from the database or from participating institutions whether transfusions occurred because of complications, as opposed to transfusion before morbidity occurred? This question is based on your observation that the incidence of transfusion increased across the risk strata that were studied. Previous studies have suggested that the increment in risk produced by transfusion is proportional to the number of units transfused. Are you able to confirm this from your data?

Did you consider preoperative anemia as a factor leading to transfusion, particularly in patients requiring more extensive preoperative evaluation and treatment? This question is suggested by your observation that myocardial infarction within a week of operation resulted in a higher incidence of transfusion, potentially related to blood testing and care of the patient before operation.

Previous studies have focused on 2 pathophysiologic mechanisms to explain morbidity and mortality associated with transfusions in the setting of cardiac surgery. One potential mechanism is impaired oxygen transport with potential cardiac, neurologic, or renal ischemia. The second purported mechanism is an increased risk of infection, presumably as a result of immune response impairment. Confirming that excess morbidity and mortality were due to these mechanisms would further validate your findings.

Finally, some of these critical issues may be settled by the RECESS trial currently in progress. This trial will test the hypothesis that the risk of transfusion is related to the storage time of transfused red blood cells.

Dr Paone. Thank you for your questions and your comments, Dr Vlahakes. I will try to go through these one at a time.

With regard to your first point, we have asked a number of our centers to provide the criteria that they use as transfusion triggers. As you might expect, there is great variation in transfusion 
triggers both during bypass and after the operation. We expected to find that with a lower intraoperative transfusion trigger, at least during bypass, those patients would be less likely to be transfused in the operating room, and we did indeed find that. In addition, as we looked at the incidence of transfusion postoperatively in these patients, we expected that they would have a somewhat higher rate, and they did. We also found that institutions that were more likely to transfuse intraoperatively were also more likely to transfuse postoperatively. So it is not all related to the trigger. I think a lot of it has to do with your approach to blood transfusion in general.

With regard to comparing transfusion rates and morbidities, we did find - at least preliminarily, and we have not concluded the analysis - that as you might also expect, as the risk level increases, so does the risk of each of the major postoperative comorbidities. Progressing from the less than $2 \%$ category to the $2 \%$ to $5 \%$ group and so on, the rate of stroke increased, the rate of wound infection increased, and so did rates of renal failure and the like. Again, we are in the process of looking more closely at these data.

As we did mention in the article and at the end of our presentation here, we don't have information yet on the volume of transfusion. Again, it is a very large database, and so there are a lot of things that we can and indeed plan to look at. A very preliminary look at volumes of transfusion again confirms what you might expect from what we already know through the literature, which is that as you increase the number of units of blood transfused to each patient, their outcomes worsen. With respect to whether this is related to the blood transfusion itself or is related to the comorbidities and the predicted risk, that analysis has not been fully completed, and we will continue to look at this issue.

The role of anemia is questionable. Many articles that have looked at these outcomes with regard to anemia or low hematocrits, particularly during bypass, have also shown relationships with mortality and transfusion rate as well. One of the criticisms of many of these studies looking at the role of hemodilutional anemia in the operating room, particularly as it relates to the worsening outcomes, is that often the analyses do not include whether the patients were transfused as well, and if so how much blood they received. If you look at the demographic variables for patients who get to lower levels of anemia, they tend to be the same exact variables that predict transfusion. So it has been difficult at best to try to discern whether it is actually the level of anemia that is causing the problem or the subsequent role of transfusion.

The mechanisms for how transfusion causes these outcomes, if indeed it does, have been widely speculated on, including those that you mentioned, immunosuppression, infection, and blood storage time and duration. Again, those are issues that with the size of the database we intend to try and look at to the best of our ability in the future.

Dr Lokeswara Rao Sajja (Hyderabad, India). I congratulate Dr Paone and colleagues on a nice presentation of a retrospective analysis. There is enough evidence that the $\mathrm{CABG}$ performed with off-pump technique has been associated with fewer homologous blood transfusions. In this analysis, was the technique of offpump surgery applied in a comparable number of patients between the 2 groups, those who received blood transfusion and those who did not receive blood transfusion?

Dr Paone. These 31,818 patients were the total population of patients undergoing CABG, including both on- and off-pump procedures. We did actually look briefly at the 2 groups. In the state of Michigan, a little more than $9 \%$ of patients underwent off-pump surgery. The transfusion rate in those cases was less than the transfusion rate for the on-pump procedures, $41 \%$ versus about $57 \%$. Interestingly, though, the mortality was about the same. The overall mortalities were $2.2 \%$ in the off-pump cases and $2.1 \%$ in the onpump cases.

We then did the analysis that we showed for the entire group for both groups separately. Although the strength of the relationship was just slightly less for the off-pump cases, the relationship itself held. When we adjusted for the predicted risk stratum, there was still a significant relationship between transfusion itself-independent of risk-and mortality in the off-pump cohort.

Dr Andrew C. Chang (Ann Arbor, Mich). Dr Paone, this study and its analysis demonstrate the strength of a collaborative effort in evaluating an important issue that crosses subspecialties in thoracic surgery. In the analysis, even though multivariate analysis identified some important factors, has your group looked at the significance of each of these factors with parsimonious model testing, such as the Akaike information criterion, to determine the goodness of fit of the final model; that is, whether the use of transfusion was as important as other comorbidities that are considered part of the PROM score?

Dr Paone. We did not. We did the propensity score analysis, and we subsequently did a standard multivariable analysis. Blood transfusion again was an independent predictor, but that was the extent of what we did.

Dr Michael Poullis (Liverpool, $U K$ ). We have repeated the exact same study you have done in a single institution with 5500 patients and found exactly the same results as you have. The one surprise finding, if you look at people who were discharged home alive, their survival was not affected by blood transfusion, which is a bit contrary to the literature. Have you looked at survival and the effect of transfusion?

Dr Paone. Thank you for your question. No, we have not. We have done some preliminary work that shows that at the lowest risk level getting small amounts of transfusion does not seem to affect survival. As I said, with the size of the database, we intend to look at that a lot more deeply and to try and determine how we can tease that out. This has been the question in this area for many years. It has been clear that there is a relationship between mortality and transfusion. It has also been clear when you look at the data that the patients who get transfusions tend to be sicker. And that is what we have tried to sort out here. I think we have sorted it out a bit, but clearly, more work needs to be done.

Dr Poullis. Yes, because I find it a little bit odd that the hazard ratio of dying with transfusion is so high-you found 4, Murphy found 6, we have 10 in Liverpool—and then as soon as you get discharged home, it goes down to 1 . It is probably the hosts more than the blood. Of course, blood is bad, but I think it's the hosts more than the blood. 